\title{
Comparisons of the Cubed-Sphere Gravity Model with the Spherical Harmonics
}

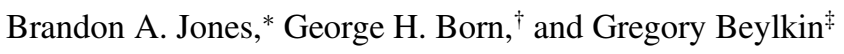 \\ University of Colorado, Boulder, Colorado 80309
}

DOI: $\underline{10.2514 / 1.45336}$

\begin{abstract}
The cubed-sphere gravitational model is a modification of a base model, e.g., the spherical harmonic model, to allow for the fast evaluation of acceleration. The model consists of concentric spheres, each mapped to the surface of a cube and combined with an appropriate interpolation scheme. The paper presents a brief description of the cubedsphere model and a comparison of it with the spherical harmonic model. The model was configured to achieve a desired accuracy so that dynamical tests, e.g., evaluation of the integration constant, closely approximate that of the spherical harmonic model. The new model closely approximates the spherical harmonic model, with propagated orbits deviating by a fraction of a millimeter at or above feasible Earth-centered altitudes.
\end{abstract}

\section{Introduction}

A LTHOUGH a sphere is an ubiquitous object, constructing a local basis on it has proven difficult. The basis functions most commonly used for a sphere are the spherical harmonics. One solution of Laplace's equation uses spherical harmonics to solve a boundary-value problem on the surface of a sphere. A solution in the spherical system of coordinates is used to construct geopotential models, such as

$$
\begin{aligned}
& U(r, \phi, \lambda)=\frac{\mu}{r}\left(1+\sum_{n=2}^{\infty} \sum_{m=0}^{n}\left(\frac{R}{r}\right)^{n} P_{n, m}[\sin \phi]\left(C_{n, m} \cos (m \lambda)\right.\right. \\
& \left.\left.\quad+S_{n, m} \sin (m \lambda)\right)\right)
\end{aligned}
$$

where $r, \phi$, and $\lambda$ are the radius, geocentric latitude, and longitude, respectively; $\mu$ is the gravitation parameter; $R$ is the radius of the primary body; $P_{n, m}$ is the associated Legendre polynomial of degree and order $n$ and $m$; and the coefficients $C_{n, m}$ and $S_{n, m}$ describe the geopotential model. Gravitational acceleration, which is required for most applications, is found by evaluating $\nabla U$.

When using the spherical harmonics, model accuracy improves by increasing the degree and order. As demand for improved gravitymodel accuracy increases, so do the computational resources required for model evaluation. Additionally, orbits about bodies with irregular mass distributions, such as the moon, require a high-degree model to properly propagate an orbit [1]. Unfortunately, an increase in the degree and order of the model by a factor of 10 results in computation time increasing by a factor of 100 [2]. Interpolation models have been developed to make evaluation faster. Some of these models preserve the spherical coordinate system $[3,4]$, while others drastically reformulate the evaluation of the gravity field $[\underline{5}, \underline{6}]$.

Presented at the 19th AAS/AIAA Spaceflight Mechanics Meeting, Savannah, GA, 8-12 February 2009; received 14 May 2009; revision received 26 October 2009; accepted for publication 7 November 2009. Copyright $(9$ 2009 by the American Institute of Aeronautics and Astronautics, Inc. All rights reserved. Copies of this paper may be made for personal or internal use, on condition that the copier pay the $\$ 10.00$ per-copy fee to the Copyright Clearance Center, Inc., 222 Rosewood Drive, Danvers, MA 01923; include the code 0731-5090/10 and $\$ 10.00$ in correspondence with the CCC.

${ }^{*}$ Graduate Student, Colorado Center for Astrodynamics Research, Department of Aerospace Engineering Sciences, 431 UCB; jonesba@ colorado.edu.

${ }^{\dagger}$ Professor, Colorado Center for Astrodynamics Research, Department of Aerospace Engineering Sciences, 431 UCB; georgeb@ colorado.edu. Fellow AIAA.

Frofessor, Department of Applied Mathematics, 526 UCB; Gregory. Beylkin@colorado.edu.
Each term of the spherical harmonic model describes a variation in the geopotential mapped over the complete sphere. For example, the $J_{2}$ term describes the equatorial deviation from a sphere for all longitudes. Hence, each term is part of a global model. Unfortunately, the spherical harmonic model is unable to meet the demands for regional representations [7]. Several alternative methods have been explored to localize the gravity field for these scientific applications [묵․

A new model, the cubed sphere, was developed to localize the representation of the gravity field and decrease the model evaluation time [2]. At its core, the cubed sphere is an interpolation model that relies on a localized representation defined on the surface of a segmented cube. We explore applications of the cubed-sphere model to orbit propagation: particularly, how it compares with the spherical harmonic model solutions.

\section{Cubed-Sphere Model}

Originally proposed by Beylkin and Cramer [2], the cubed-sphere model defines a new method to compute geopotential and acceleration. Essentially, the sphere is mapped to a cube with a new coordinate system defined on each face. Each face is segmented by a uniform grid, and interpolation is performed to find the acceleration. Multiple spheres, each mapped to a cube, are nested within each other, and interpolation is performed between adjacent shells to account for the acceleration variation in the radial direction. The mapping of a sphere to a cube is illustrated in Fig. 1. A grid-spacing scheme is established, with values for acceleration precomputed at intersections of the grid lines. Basis splines, or B-splines, were selected to represent functions on each face of the cube. The next subsection describes the cubed-sphere model in detail.

Three models were proposed in [2]: 1) B-splines defined on the surface of a sphere, 2) polynomials on subdivisions of the surface of the cube, and 3) B-splines on the surface of a cube. This study uses the third model, which combines the benefits of the first two. In option 1, defining B-splines on the surface of the sphere maintains the stretching and the resulting oversampling near the poles. In option 2, using piecewise polynomials on the face of the cube requires a higher sampling rate than using splines. In option 3, using splines on the faces of the cube does not have stretching near the poles associated with the first model and uses the lower sampling rate associated with splines. Hence, option 3 is more efficient for our purposes.

A major goal of the cubed-sphere model development is to improve computational efficiency when using geopotential models. Effectively, it is a trade of speed for memory, i.e., file size. However, a modern computer has more than the sufficient memory for this purpose. 


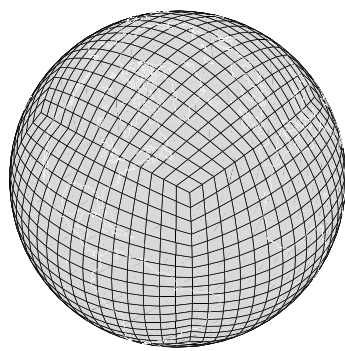

Fig. 1 Illustration of the mapping from a sphere to a cube.

\section{Model Description}

The cubed-sphere model may be used to approximate any number of elements defined on a primary body. For example, it can approximate each component of acceleration, or the gravity potential. The accelerations are not directly derived from the potential, but are stored separately (in a submodel). Thus, for a model to provide both potential and three components of acceleration, values of all four parameters are stored at each point for future interpolation. In the following sections, any reference to modeling the gravity potential may also be applied to modeling acceleration (with the appropriate adjustments). Although the cubed-sphere model has been described in the literature [2], a more detailed description is included here for the purpose of clarity.

The cubed-sphere model is currently derived from an existing gravity model, hereafter called the base model. Although other models such as a polyhedron or mascon may be used, we currently use the spherical harmonic model as the base model. In the cubed-sphere model, the first four terms of the spherical harmonic expansion [i.e., the two-body term, $J_{2}$, and the $(*)_{2,1}$ and $(*)_{2,2}$ terms] are used directly. The cubed-sphere model does not include the lower-order terms to reduce the range of approximated values, reducing the cost of maintaining accuracy in the local model. The geopotential values computed by the remaining terms in the base model are then represented by the basis functions on the surface of the cube.

Temporal variations, such as solid or liquid tides, influence the geopotential. These variations mostly affect lower-degree terms of the potential. The cubed sphere only models terms of degree greater than or equal to a chosen minimum degree and order: in this case, 3 . This parameter may be adjusted to allow for perturbations in the lower-degree terms, whereas higher-degree terms are expressed in the cubed-sphere formulation. Of course, this may slightly affect computation time.

A key parameter of the cubed-sphere model is the grid size $N$. Similar to the degree and order of the spherical harmonic model, the grid size defines the density of the grid on each cube face and is a measure of model fidelity. For a given altitude, the values of latitude and longitude are segmented such that

$$
\lambda=2 \pi x, \quad \phi=2 \pi y
$$

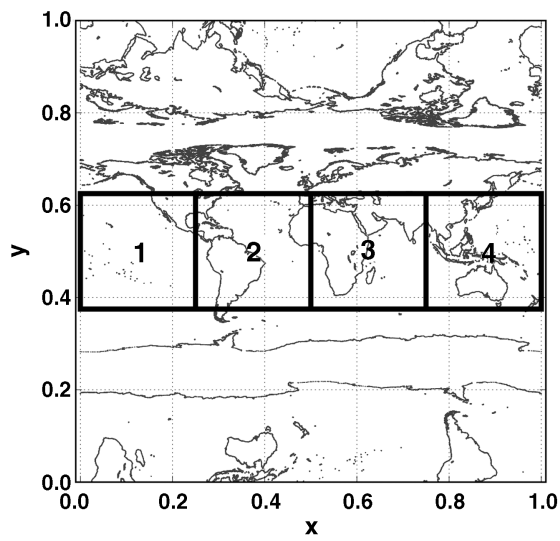

Fig. 2 Illustration of the mapping of the Earth surface to an $x-y$ plane to make $U_{p} 2 \pi$-periodic. Selected portions form the cubed sphere. The second plot illustrates the process for the rotated coordinate system.

where $x$ and $y$ are discrete values in the range $[0,1)$ with spacing $N^{-1}$. To have a fast algorithm to compute coefficients of the B-splines from the values of the spherical harmonic model, the values of the latitude $\phi$ are extended to the interval $[0,2 \pi)$. It may not be readily apparent how this is done, but this will be explained in a moment.

Latitude and longitude are mapped to a two-dimensional grid specified by $x$ and $y$ to solve for the B-spline coefficients. As described in the Appendix, the interpolation coefficients are easily computed in the Fourier domain. Since the grid variables $x$ and $y$ are 1-periodic, we may use the two-dimensional fast Fourier transform (FFT) algorithm to provide a fast method for finding the B-spline coefficients.

If $\phi$ only varies from $-\pi / 2$ to $\pi / 2$ or from 0 to $\pi$, then $y$ is not 1-periodic and the FFT algorithm cannot be used directly. To periodically extend the Earth's geopotential, we duplicate it to complete the period. The mathematical formulation of the new geopotential $U_{p}$ is then

$$
U_{p}(r, \phi, \lambda)= \begin{cases}U(r, \phi, \lambda) & \text { if } 0 \leq \phi<\pi \\ U(r, 2 \pi-\phi, \lambda+\pi) & \text { if } \pi \leq \phi<2 \pi\end{cases}
$$

and $\phi$ is now a value in the range $[0,2 \pi)$. Thus, $U_{p}$ is $2 \pi$-periodic. The offset of the longitude by $\pi$ assures that all spherical harmonics are extended smoothly (with all derivatives) to the interval $[0,2 \pi$ ). One can see this by the fact that one period of $\phi$ circumscribes the primary body. Thus, $U_{p}$ is infinitely differentiable in both variables so that the trapezoidal rule can be used to discretize the Fourier integrals. The FFT algorithm may be used directly to compute the B-spline coefficients. Note that the doubling of the geopotential model is only used to generate these coefficients. To prevent grid distortion given the ambiguity of longitude at the poles, the coordinate system is rotated so that the poles lie along the equator, which is equivalent to using the transverse Mercator map projection. A second $x-y$ plane is generated after this rotation, with the FFT algorithm applied and a second set of B-spline coefficients determined. B-spline coefficients are defined over the surface of the two $x-y$ grids, which are then broken into appropriate segments to generate the faces of a cube. Each cube face has a new $x-y$ grid with axes defined over the range $[-1,1]$. Four segments along the middle latitudes are selected from the first set of coefficients, with each segment corresponding to a cube face. The two remaining faces at the poles are selected from the second set. This process is illustrated in Fig. 2.

Although grid spacing is preserved along the face of the cube, we note that the grid size on each face is $N / 4$ by $N / 4$. This parameter is used in the naming convention for a given model. A CS- $X$ model is a cubed-sphere model, where $X$ corresponds to the grid size on a cube face, or $N / 4$. To summarize, we have described the geopotential model at a given altitude on the surface of a cube.

Since the spherical harmonics is a global model, a uniform grid density is forced over the complete surface to model the highfrequency variations. Of course, a true Earth model will have

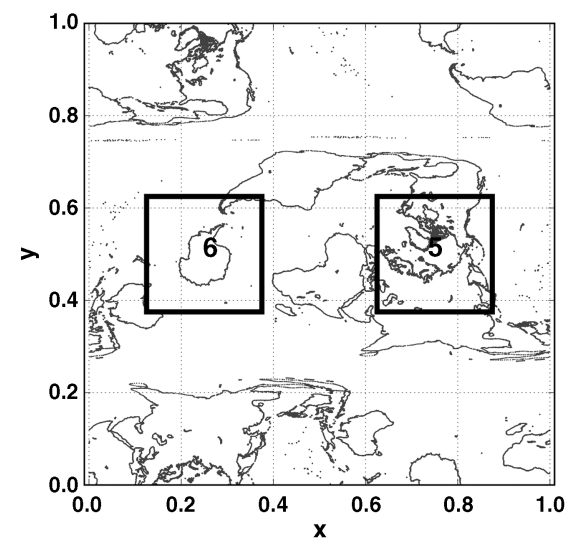



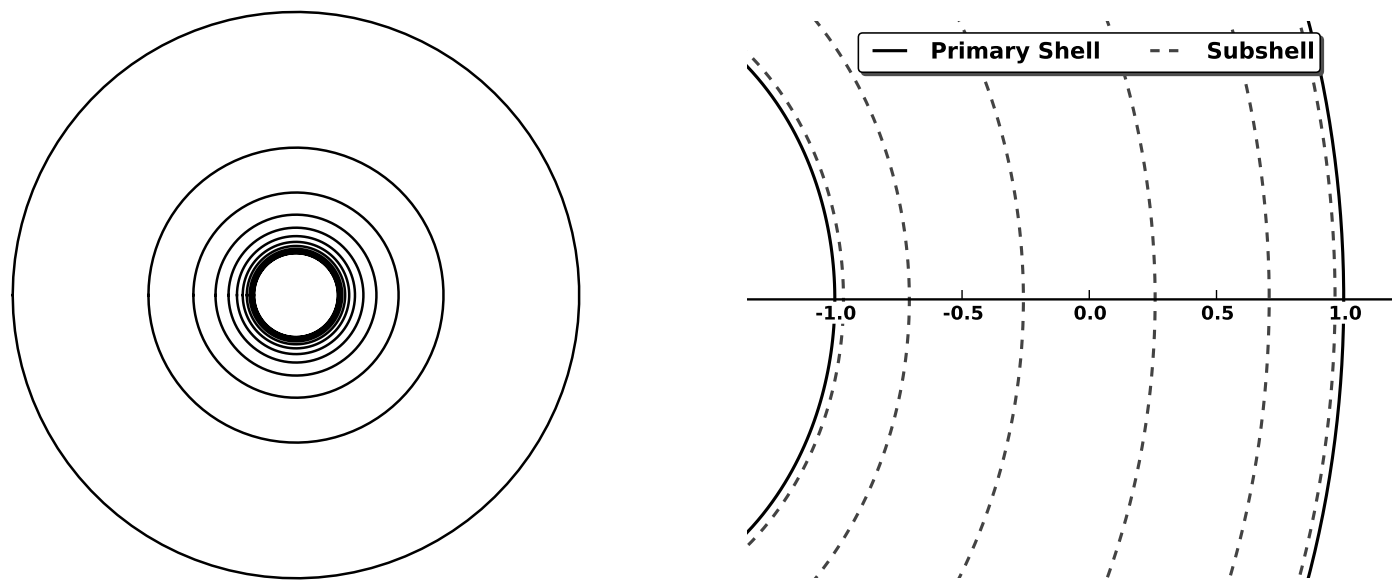

Fig. 3 Illustration of the increased density of primary shells with closer proximity to the primary body (left figure) and an example of the subshell spacing between primary shells (right) for the cubed sphere.

relatively large variations in the gravity field as location changes. This is especially true along coastal and mountain regions. As discussed in [2], the cubed sphere was originally developed with multiresolution techniques in mind. However, adjusting the grid density to these levels reveals noise in the original spherical harmonics terms. The noise could be removed, which effectively modifies some higher-degree terms. Thus, the cubed sphere would no longer agree with the spherical harmonic model, which may currently cause resistance to its use. Additionally, early tests of the model for [2] demonstrated only small gains in speed as a result of such change and only a marginal decrease in memory required.

A user-specified number of nested concentric shells are required for interpolation in the radial direction. Shell spacing is determined by defining a set number of points $\left(h_{j}\right)$ equally spaced in the interval $[0,1]$. Shell locations are then

$$
\frac{R}{r_{j}}=1-h_{j}^{2}
$$

where $r_{j}$ is the radial distance of the spherical shell. We note that as the shell radius $r_{j}$ approaches infinity, $R / r_{j}$ tends to 0 . Shell density increases for lower altitudes, corresponding to the inverse square relationship between geopotential and radius. This is illustrated in Fig. 3. The final shell at infinity is used only as a boundary for determination of the subshells.

The primary shells each consist of subshells for interpolation in the radial direction. The cubed sphere approximates the potential using Chebyshev polynomial interpolation of degree $l$ :

$$
U(x)=\sum_{i=0}^{l} a_{i} T_{i}(x)
$$

where $a_{i}$ are (spatially dependent) coefficients and $T_{i}$ are Chebyshev polynomials, values of which are computed via the three-term recurrence:

$$
\begin{gathered}
T_{0}(x)=1 \\
T_{1}(x)=x \\
T_{i}(x)=2 x T_{i-1}(x)-T_{i-2}(x)
\end{gathered}
$$

The space between subshells is mapped to the range $[-1,1]$, where zero corresponds to the midpoint between primary shells. The subshells are then positioned at the roots of the $l+1$ degree Chebyshev polynomial (the so-called Gaussian nodes). This is illustrated in Fig. 3 for a fifth-degree Chebyshev scheme with six subshells. The Gaussian nodes are selected to minimize interpolation error (see, for example, [10]). In generation of the cubed-sphere model, each subshell is independent of all others. B-spline coefficients for each subshell are generated as previously described using the applicable altitude for the evaluation of Eq. (3). A total of $(l+1) \times(M-1)$ subshells are computed, where $M$ is the number of primary shells. It is important to note that the mapping to the cube is simply used for data storage and finding the spline coefficients. All values described by the model (more specifically, components of acceleration) are still represented in the spherical coordinate system. The cube provides a uniform grid for B-spline coefficients and a data structure for faster computation.

Grid density is kept constant for all subshells at all altitudes. Since the high-frequency variations in the spherical harmonics attenuate quickly with altitude, the required grid density for higher altitudes is less than those required closer to the planet. This has not been currently integrated with the cubed sphere, but is a topic of current research. In [11], the authors are seeking an efficient relationship between the required grid density and altitude to meet accuracy requirements. Results of this research may also be incorporated into the cubed sphere.

Implementation of the cubed-sphere model is fairly straightforward. Lower-degree components of geopotential are computed directly using the spherical harmonic coefficients stored in the model. For higher-degree terms, the point of interest is mapped to the cube. The value is obtained by the interpolation on the $l+1$ subshells within the primary shell containing the point of interest. The coefficients required for Eq. (5) are then

$$
a_{i}=\frac{2-\delta_{i 0}}{l+1} \sum_{j=0}^{l} U_{j} T_{i}\left(x_{j}\right)
$$

where $U_{j}$ is the potential on the $j$ th subshell at $x_{j}$, and $\delta_{i j}$ is the Kronecker delta function.

The conversion from spherical $(\phi, \lambda)$ to cube coordinates $(x, y, n)$ is summarized in Table 1 and was adapted from [2], with some simplifications and corrections of misprints. For the cube coordinates, $n$ refers to the face of the cube. The first four faces represent the sphere at the middle latitudes between $-\pi / 4$ and $\pi / 4$, and faces 5 and 6 correspond to the polar regions. For faces $1-4$, the

Table 1 Spherical to rectangular coordinates conversion.

Note $\gamma=-\cos \phi \sin \lambda, \omega=\tan \phi / \cos \lambda$, and $\alpha=4 / \pi$

\begin{tabular}{lccc}
\hline \hline Face & Angle ranges & $X$ coordinate & $Y$ coordinate \\
\hline 1 & $-\pi \leq \lambda<-\pi / 2$, & $\alpha \lambda+3$ & $\alpha \phi$ \\
& $-\pi / 4 \leq \phi \leq \pi / 4$ & & \\
2 & $-\pi / 2 \leq \lambda<0,-\pi / 4 \leq \phi \leq \pi / 4$ & $\alpha \lambda+1$ & $\alpha \phi$ \\
3 & $0 \leq \lambda<\pi / 2,-\pi / 4 \leq \phi \leq \pi / 4$ & $\alpha \lambda-1$ & $\alpha \phi$ \\
4 & $\pi / 2 \leq \lambda<\pi,-\pi / 4 \leq \phi \leq \pi / 4$ & $\alpha \lambda-3$ & $\alpha \phi$ \\
5 & $\phi>\pi / 4$ & $\alpha \tan ^{-1}(\omega)-2$ & $\alpha \sin ^{-1}(\gamma)$ \\
6 & $\phi<-\pi / 4$ & $\alpha \tan ^{-1}(\omega)+2$ & $\alpha \sin ^{-1}(\gamma)$ \\
\hline \hline
\end{tabular}


Table 2 Cubed-sphere configurations. The original formulation refers to [2], the second formulation refers to this paper

\begin{tabular}{lcc}
\hline \hline Property & $\begin{array}{c}\text { Original } \\
\text { formulation }\end{array}$ & $\begin{array}{c}\text { Current } \\
\text { formulation }\end{array}$ \\
\hline Number of values estimated $P$ & 3 & 4 \\
B-spline degree $m$ & 11 & 11 \\
Chebyshev polynomial degree & 5 & 11 \\
Number of primary shells $M$ & 14 & 14 \\
Grid Density $N$ & & \\
CS-30/20 $\times 20$ & 80 & 120 \\
CS-76/70 $\times 70$ & 280 & 304 \\
CS-162/150 $\times 150$ & 600 & 648 \\
\hline \hline
\end{tabular}

conversion to the $x-y$ coordinate system is simply a map from the appropriate angle range $\{$ for example, $[-\pi, \pi / 2)$ to $[-1,1)\}$. The mappings for faces 5 and 6 result from rotating the sphere so that the poles lie on the equator. The original $\phi$ and $\lambda$ values are converted to Cartesian coordinates and are then rotated about the $x$ axis by $-\pi / 2$. The new Cartesian coordinates are converted to a new $\phi^{\prime}$ and $\lambda^{\prime}$ and mapped to the range $[-1,1)$ in a manner similar to that for faces $1-4$. The equations for faces 5 and 6 in Table 1 are derived from this procedure.

\section{Model Configuration}

Test software was written to evaluate the model at a number of random points above the primary body. For each point, the cubedsphere model is compared with the base model and the absolute deviation is calculated. The maximum deviation for all points is then used to characterize the accuracy of the model. Tests used $10^{5}$ random points. Any increase in the number of points has been yielding similar results. The acceleration computed via the original cubed-sphere model (developed for [2]) differed from that of the spherical harmonic model by as much as $10^{-9} \mathrm{~m} / \mathrm{s}^{2}$. Although this error may appear rather small, the current model was reconfigured, resulting in improvement of the accuracy to $10^{-11} \mathrm{~m} / \mathrm{s}^{2}$ for high-fidelity models. The main focus of this reconfiguration was to improve model performance for higher-fidelity models, i.e., equivalents to the $70 \times$ 70 models and those of higher degree or order.

The original model configuration was selected to meet Nyquist rate requirements; the grid spacing equaled half the spatial resolution described by the given spherical harmonic base model. This new configuration was empirically determined to meet the desired accuracy agreement with the base model. Full benefits of the reconfiguration are demonstrated in the section discussing the integration-constant performance.

Changes to the cubed-sphere configuration made in the course of this research, along with other key model parameters, are included in Table 2. Note that the change in Chebyshev polynomial degree and grid densities did increase the file size. Additionally, the original model was constructed for three acceleration values and did not include the estimate of the potential. These configuration parameters may be tailored to a specific design based on orbit accuracy requirements and file-size limitations. If the potential is not required, the file size is reduced by almost $25 \%$. Table 2 defines the base models for the CS-30, CS-76, and CS-162 models used throughout this study. Other changes were made to the software to improve computational speed and switch from the unnormalized to normalized formulation of the associated Legendre functions in the base model. Finally, the model storage scheme of the B-spline coefficients was altered to allow for loading of selected shells to reduce software memory requirements and decrease the initialization time.

The number of elements that must be stored in the model are estimated using

$$
\text { number of elements }=6 P(l+1)(M-1)\left(\frac{N}{4}+m\right)^{2}
$$

where the meaning of the terms is described in Table 2. Additionally, there is some memory overhead associated with the file header. For the CS-30 model, all of this results in a 49 MB file. Similarly, an $856 \mathrm{MB}$ file is required for a CS-162 model. Since the model is localized, only the subshells required for a given orbit need to be generated or loaded into memory.

The B-spline coefficients are organized such that no search is necessary. If the degree of the interpolating functions remains constant for each grid size, model evaluation time remains constant. Experimental results demonstrate that the evaluation time of the cubed-sphere model is slightly more than that for the $20 \times 20$ spherical harmonic models. Unlike with the spherical harmonic model, as the degree and order increase, resulting in higher grid density, the cubed-sphere evaluation time remains constant. Thus, speedup factors compared with the spherical harmonics rapidly increases with model fidelity.

Continuity of the model determined values may be a concern for some applications. Primary shells may be generated to overlap. This has not yet been explored, but current research into developing improved models $[11,12]$ would perhaps eliminate this need. Given the lack of overlap in the primary shells, model discontinuities may exist at the primary shell boundary. However, this new configuration reduces these discontinuities to within the model noise. Specifically, the discontinuities are less than the machine precision for the given acceleration values. Discontinuities do not exist between faces of the cube, since the FFT algorithm used to solve for the interpolation coefficients uses the entire gravity field at the given altitude.
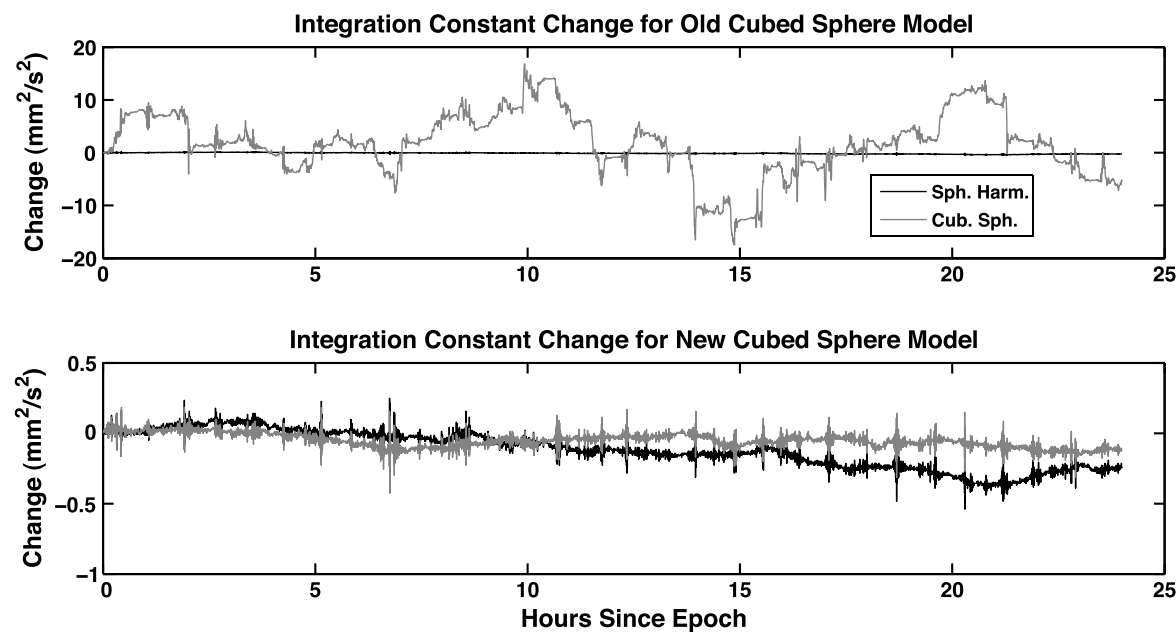

Fig. 4 Changes in integration constant with the new CS-162 model configuration versus the configuration of [2] for an orbit with an inclination of $15^{\circ}$ and right ascension of $50^{\circ}$ at a $300 \mathrm{~km}$ altitude. 
Table 3 Percentage of runs where $\mathcal{O}\left(K-K_{\circ}\right)$ is less than the other model

\begin{tabular}{lcc}
\hline \hline Model & Cubed sphere & Spherical harmonics \\
\hline CS-30 & $0.024 \%$ & $0.020 \%$ \\
CS-76 & $0.012 \%$ & $0.008 \%$ \\
CS-162 & $0.264 \%$ & $0.272 \%$ \\
\hline \hline
\end{tabular}

\section{Comparisons to the Spherical Harmonic Model}

After the cubed-sphere reconfiguration, we compared it with the spherical harmonic model. The GGM02C [13] model was selected as both the base model of the cubed sphere and the basis of comparison for the following tests. Evaluations included a comparison of the integration constant, spatial comparisons of the models in the form of gravity anomalies, and the propagated orbits themselves.

Using the TurboProp orbit-integration package [14] minimized software development time. This software provides integration tools implemented in $\mathrm{C}$ that are compatible with MATLAB and Python. The cubed-sphere model, along with the necessary interface code, was implemented within the TurboProp framework. However, the software may be easily ported to other packages. Tests requiring orbit propagation used the TurboProp Runge-Kutta 7(8) integrator with an integration tolerance of $10^{-12}$. Other integrators were tested, including symplectic Runge-Kutta algorithms, and yielded similar results.

For test orbits, we employed a variety of initial conditions and output states every $20 \mathrm{~s}$ over the $24 \mathrm{~h}$ propagation. The initial orbit altitude spanned 100 to $1000 \mathrm{~km}$ at $50 \mathrm{~km}$ intervals. Cubed-sphere model accuracy relative to that of the spherical harmonic model decreases at lower altitudes. However, since most satellites orbit at or above $300 \mathrm{~km}$, we provide altitude-specific results at $300 \mathrm{~km}$. The right ascension of the ascending node $(\Omega)$ ranged from 0 to $180^{\circ}$ in $5^{\circ}$ increments, and the inclination $i$ varied from 0 to $85^{\circ}$ in $2.5^{\circ}$ intervals. All other orbit elements were initially set to zero. The maximum inclination of $85^{\circ}$ avoids the singularity at the poles in the classical formulation of the spherical harmonic model. Thus, for each altitude, 1295 orbits were tested. The Greenwich sidereal time was set to $0^{\circ}$ at the epoch time, with an Earth rotation rate of $360^{\circ}$ per solar day. The planetary radius and gravitation parameter were set to the appropriate values as determined by the base model. Each set of initial conditions was propagated using the cubed-sphere model and the corresponding base model. The trajectories were compared and 3-D rms differences were calculated and stored. An orbit-propagation error refers to the difference between the cubedsphere and spherical harmonic model orbits. Tests consisted of three models and are described in Table 2 .

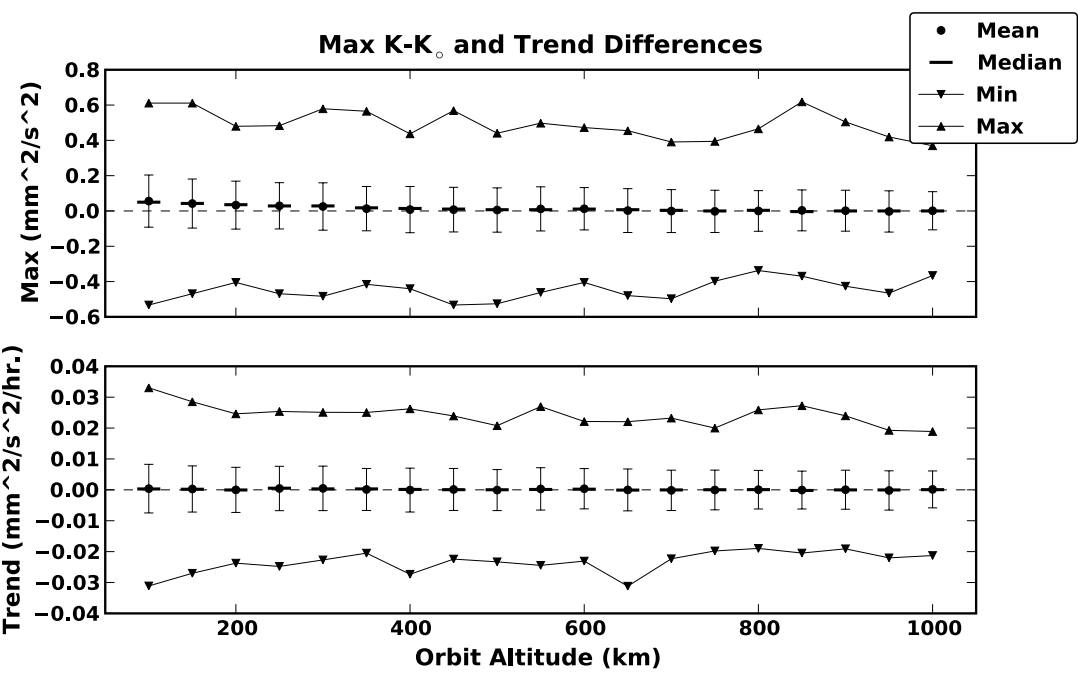

Fig. 5 Comparison of the integration-constant variations for the CS-30 model with the spherical harmonic base model. Error bars are 1- $\sigma$.
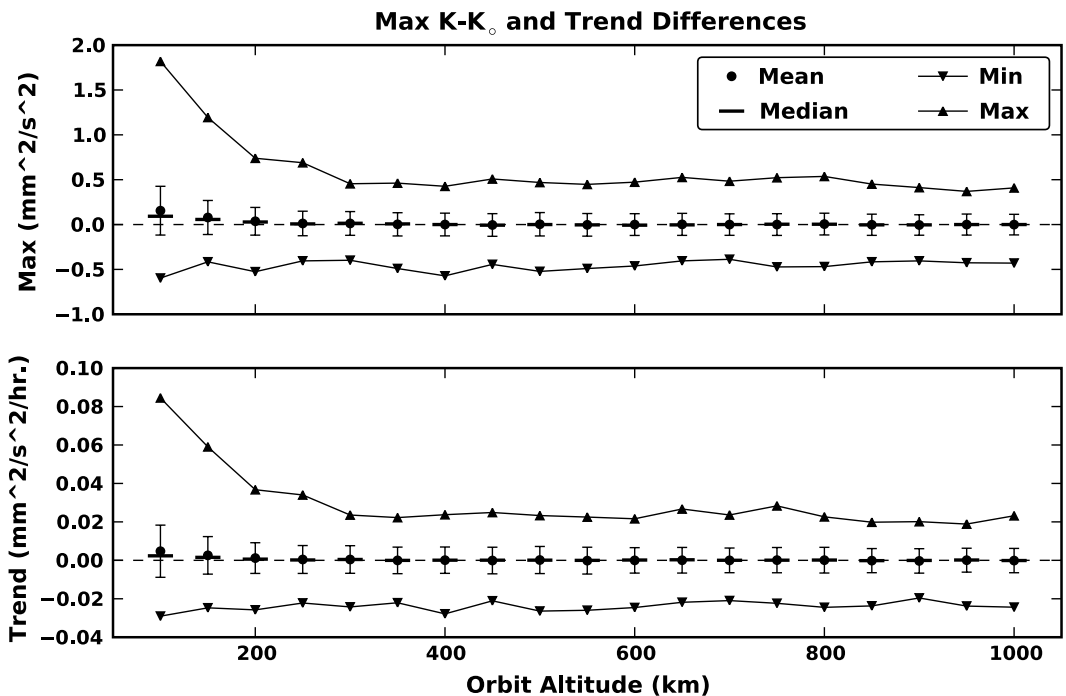

Fig. 6 Comparison of the integration-constant variations for the CS-76 model with the spherical harmonic base model. Error bars are 1- $\sigma$. 



Fig. 7 Comparison of the integration-constant variations for the CS-162 model with the spherical harmonic base model. Error bars are 1- $\sigma$.

Integration (Jacobi) Constant Comparisons

A given geopotential model must satisfy the Laplace equation:

$$
\nabla^{2} U=\frac{\partial^{2} U}{\partial x^{2}}+\frac{\partial^{2} U}{\partial y^{2}}+\frac{\partial^{2} U}{\partial z^{2}}=0
$$

Unfortunately, the direct calculation of the second derivatives within the cubed-sphere model from accelerations results in the loss of accuracy of one-two digits. To avoid such a loss of accuracy, values of the derivatives may be added to the model based on the variational equations of the spherical harmonic model. (Of course, this will almost double the file size.)

Instead of testing the cubed sphere under the Laplace criterion, this study applies another technique using the Jacobi-like integration constant [15]:

$$
K=\frac{\dot{\mathbf{r}} \cdot \dot{\mathbf{r}}}{2}-\left[\frac{\mu}{r}-U(\mathbf{r}, t)\right]-\omega \cdot(\mathbf{r} \times \dot{\mathbf{r}})
$$

This constant assumes that the geopotential is time-varying (which is valid, due to Earth rotation). Here, $\omega$ is the angular velocity of the primary body. For a valid gravity model and a propagated orbit, $K$ must remain constant over time, or $K-K_{\circ}$ equals zero. In practice, the constant fluctuates due to the numerical integration process and errors in the estimate of the gravity field.

As mentioned previously, the cubed-sphere reconfiguration improves accuracy: specifically, the integration-constant performance. Figure 4 illustrates the extent of the improvement. Previously, the cubed-sphere integration-constant deviations were consistently one-three orders of magnitude greater. This would have resulted in reducing the validity of the model for applications requiring longterm orbit propagation. In some cases, such as this example, the integration-constant test for the cubed sphere performs even better than the spherical harmonic model.

A major concern when comparing the variations in the integration constant for the cubed-sphere and the spherical harmonic models is the relative magnitude of the fluctuations. In some cases, the magnitude of the variations of the cubed-sphere model were as much as an order of magnitude less than the spherical harmonic model, and vice versa. Table $\underline{3}$ provides the percentage of the 24,605 runs for each model that exhibited this behavior. In most cases, the orders of magnitude of the fluctuations were the same. However, a small percentage of the tests yielded integration-constant changes an order of magnitude less for one model when compared with the other. In the case of the CS-162 model, where the percentage of runs sharply increased, tests at altitudes at or below $250 \mathrm{~km}$ exhibited the larger fluctuations.

In Fig. 5, we illustrate the performance of the CS-30 model under the integration-constant test as compared with the spherical harmonic model. The top figure depicts the relative performance of the maximum fluctuations. For the comparison of the fluctuation magnitude, the maximum absolute deviations for both the cubedsphere and the spherical harmonic model orbits are differenced. We compute the mean, median, min, max, and standard deviations of these differences for all orbits at a given altitude and illustrate these values for all altitudes tested. Note that a negative number indicates that the integration-constant fluctuations for the cubed-sphere model orbit are less than those of the spherical harmonic model orbit. The

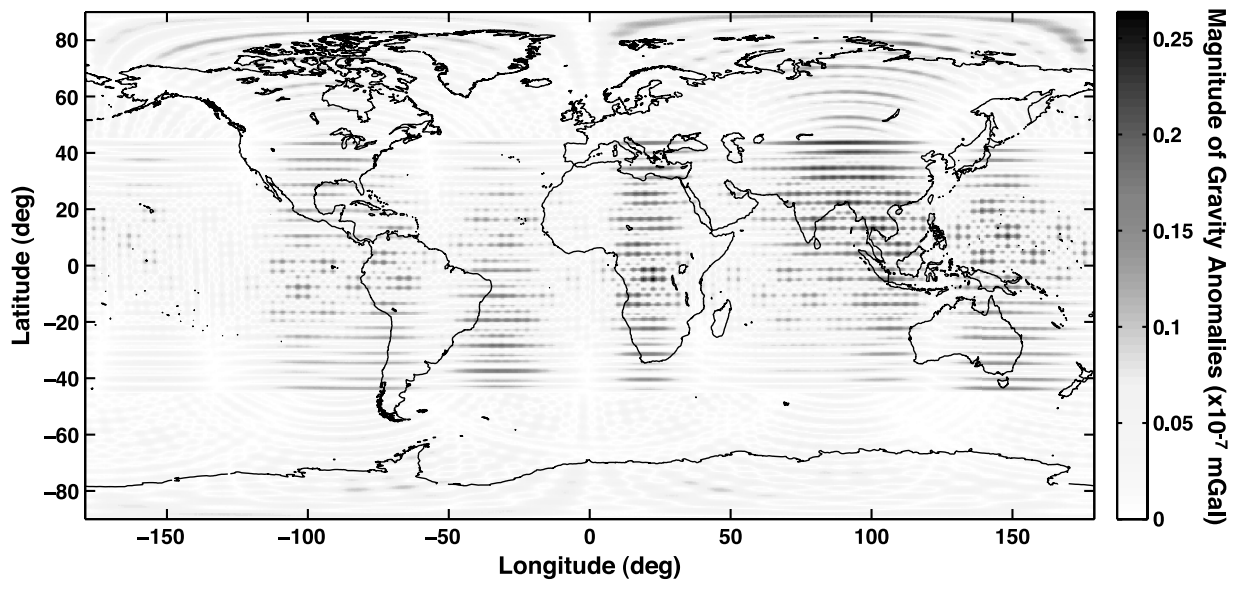

Fig. 8 CS-30 gravity anomalies at $300 \mathrm{~km}$. 


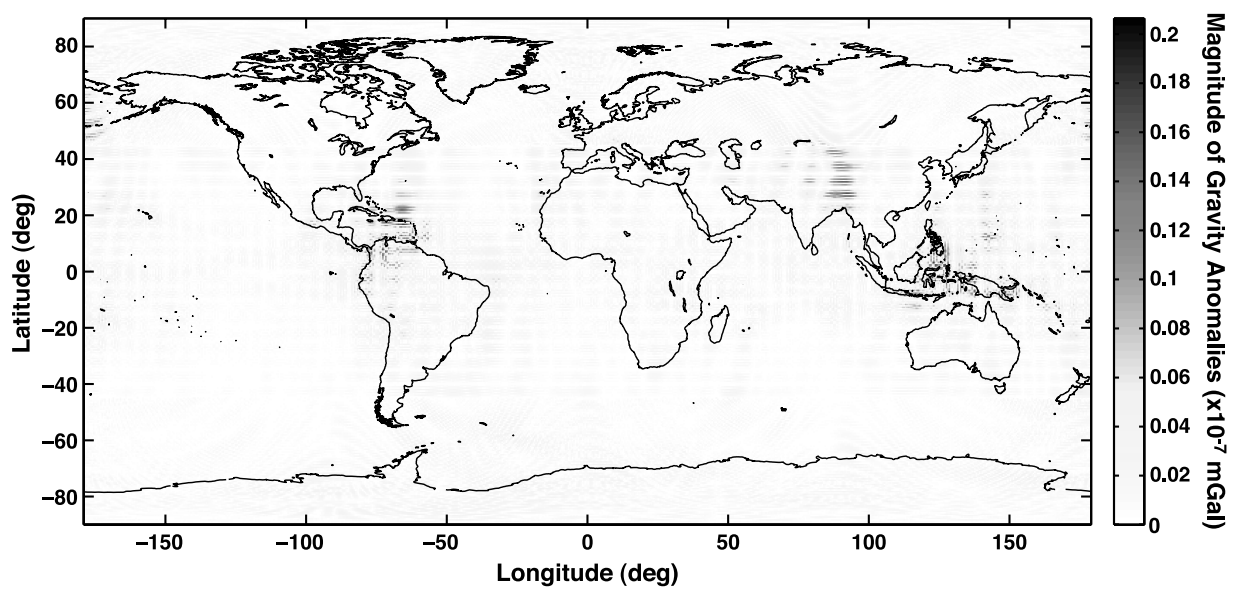

Fig. 9 CS-162 gravity anomalies at $300 \mathrm{~km}$.

bottom subplot portrays the relative performance of trends in the integration-constant variations. For each orbit, we perform a linear fit to the integration-constant fluctuations. The trend in the fluctuations is described by the slope, with Fig. 5 illustrating the differences in these trends. For the slope in the trend line, units are designated as $\mathrm{mm}^{2} / \mathrm{s}^{2} / \mathrm{h}$, since the units of the integration constant are $\mathrm{mm}^{2} / \mathrm{s}^{2}$ (i. e., change in the constant per hour). For altitudes below $400 \mathrm{~km}$, the mean and median magnitude differences indicate that the spherical harmonic model slightly outperforms the cubed sphere. However, the average difference drops to nearly zero above $400 \mathrm{~km}$. The maximum and minimum differences remain consistent. Given that the mean and median differences in the trend line slope are around zero with 1- $\sigma$ values within $0.01 \mathrm{~mm}^{2} / \mathrm{s}^{2} / \mathrm{h}$, the two models typically have the same long-term trend.

Similarly, Fig. 6 illustrates the integration-constant performance for the CS-76 model. Note that the magnitude and trend of the integration-constant change is larger for the cubed sphere at lower altitudes, but settles at around $300 \mathrm{~km}$. The median is smaller than the
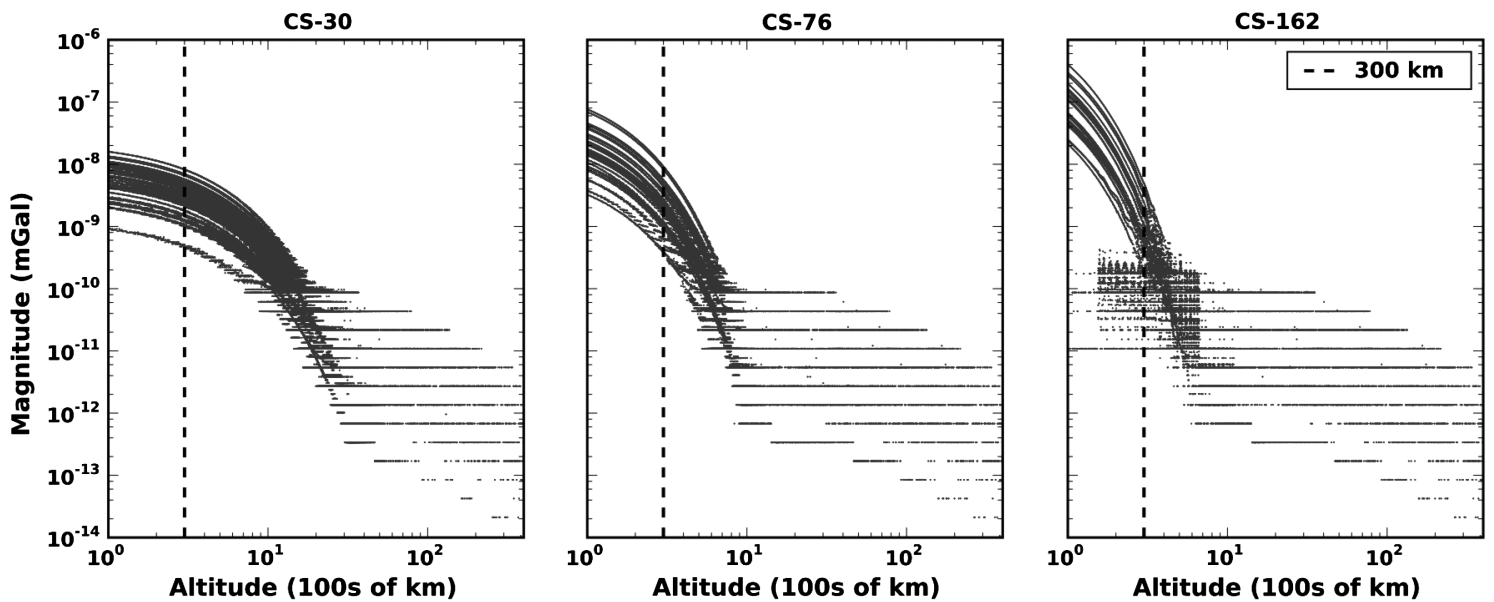

Fig. 10 Variations in gravity anomalies with altitude for 42 points on the Earth using the CS-30, CS-76, and CS-162 models.
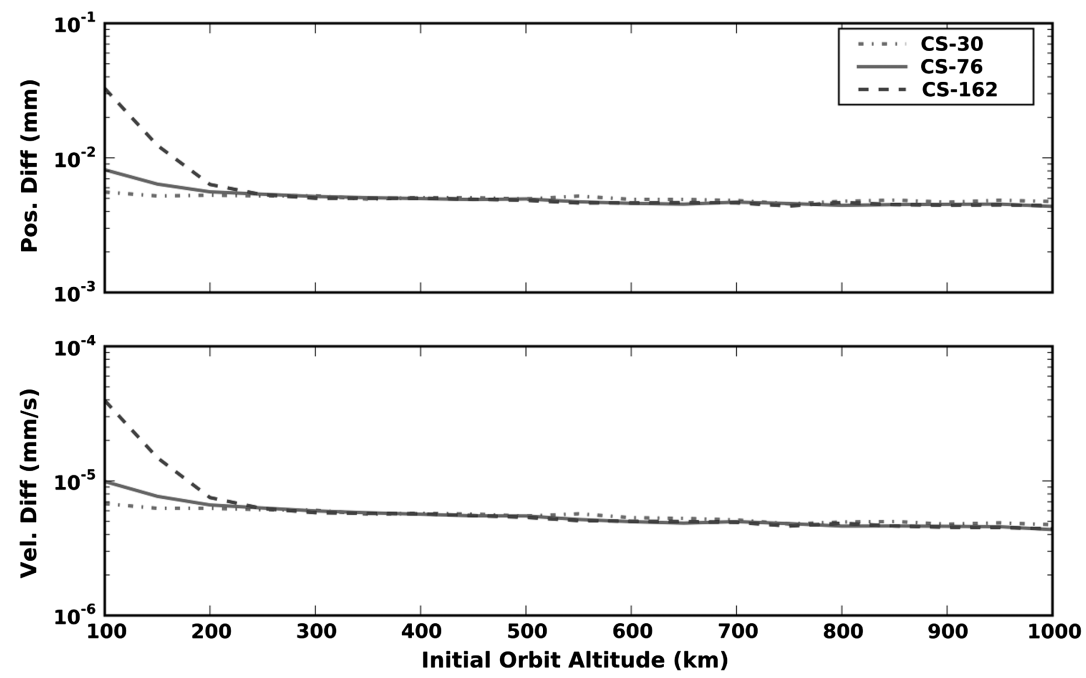

Fig. 11 Summary of the total 3-D rms orbit-propagation differences for the CS-30, CS-76, and CS-162 models at a given initial altitude. 
Table 4 Average speedup factor for the cubed-sphere versus the base model

\begin{tabular}{lcc}
\hline \hline $\begin{array}{c}\text { Cubed-sphere } \\
\text { model }\end{array}$ & $\begin{array}{c}\text { Spherical harmonic } \\
\text { model }\end{array}$ & $\begin{array}{c}\text { Average speedup } \\
\text { factor }\end{array}$ \\
\hline CS-30 & $20 \times 20$ & 0.73 \\
CS-76 & $70 \times 70$ & 5.97 \\
CS-162 & $150 \times 150$ & 30.82 \\
\hline \hline
\end{tabular}

mean at these lower altitudes, indicating a relatively small number of tests increase the mean value. Again, the models closely agree for higher altitudes, as indicated by the mean and median values with small error bars.

Results for the CS-162 model are provided in Fig. 7. Note that some extreme values have been truncated to improve visibility of performance statistics at higher altitudes. In the case of the differences in the magnitude differences, the minimum values for the 100 and $150 \mathrm{~km}$ orbits are -3.39 and $-1.51 \mathrm{~mm}^{2} / \mathrm{s}^{2}$, respectively. The maximum values are 5.20 and $1.79 \mathrm{~mm}^{2} / \mathrm{s}^{2}$. In the case of the trend slope differences, the missing maximums are 0.44 and $0.14 \mathrm{~mm}^{2} / \mathrm{s}^{2} / \mathrm{h}$. Like the CS-76 model, differences between the cubed-sphere and spherical harmonic models are greater at lower altitudes. This trend remains consistent through the remaining tests and is attributed to the greater differences in the gravity anomalies at lower altitudes seen in the next section. In this case, the differences in the models settle around $250 \mathrm{~km}$.

\section{Gravity Anomaly Comparisons}

This section provides a comparison of the spatial differences in the acceleration. The results are represented as gravity anomalies projected onto the surface of the Earth. The acceleration vector was evaluated at a common altitude with latitude and longitude varied in $0.5^{\circ}$ intervals. The magnitude of the differences in the vector is then illustrated in Figs. $\underline{8}$ and $\underline{9}$. Like in the orbit-propagation tests, acceleration vectors were not computed at the poles to avoid the singularity present in the spherical harmonic model.

Figure 8 illustrates the gravity anomalies for the CS-30 model at an altitude of $300 \mathrm{~km}$ and shows that anomalies are less than $10^{-7} \mathrm{mGal}$. The cube grid is visible, for example, over the East Indies. The regions of peak variation correspond to regions of high gravity fluctuations, as determined by the spherical harmonic model (e.g., the Himalayan mountain ranges and the East Indies). We note that the GGM02C gravity model is not accurate to $10^{-7} \mathrm{mGal}$, and the gravity anomalies in Figs. $\underline{8}$ and $\underline{9}$ are within the error of the base model.

The gravity anomalies for the CS-162 model at $300 \mathrm{~km}$ are found in Fig. 9. The grid spacing is still visible, but areas of peak variations are isolated to select regions. Like the previous cases, these regions correspond to those with a generally large variation in the gravity field. The magnitude of the anomalies is again on the order of $10^{-8} \mathrm{mGal}$, with the largest anomaly off of the coast of Haiti at approximately $18^{\circ}$ latitude and $-67^{\circ}$ longitude. Gravity anomalies for the CS-76 model are not included, but yield similar results to those in the previous two models. The grid spacing is also visible, but the regions of higher differences were not as isolated as the CS-162 case.

To illustrate gravity anomaly variations with altitude, Fig. 10 depicts the difference in gravity anomalies for each of the models at altitudes up to geosynchronous orbit. Most of the 42 points depicted were randomly selected, although we chose a couple of points to coincide with regions of large anomalies. As expected, peak variations
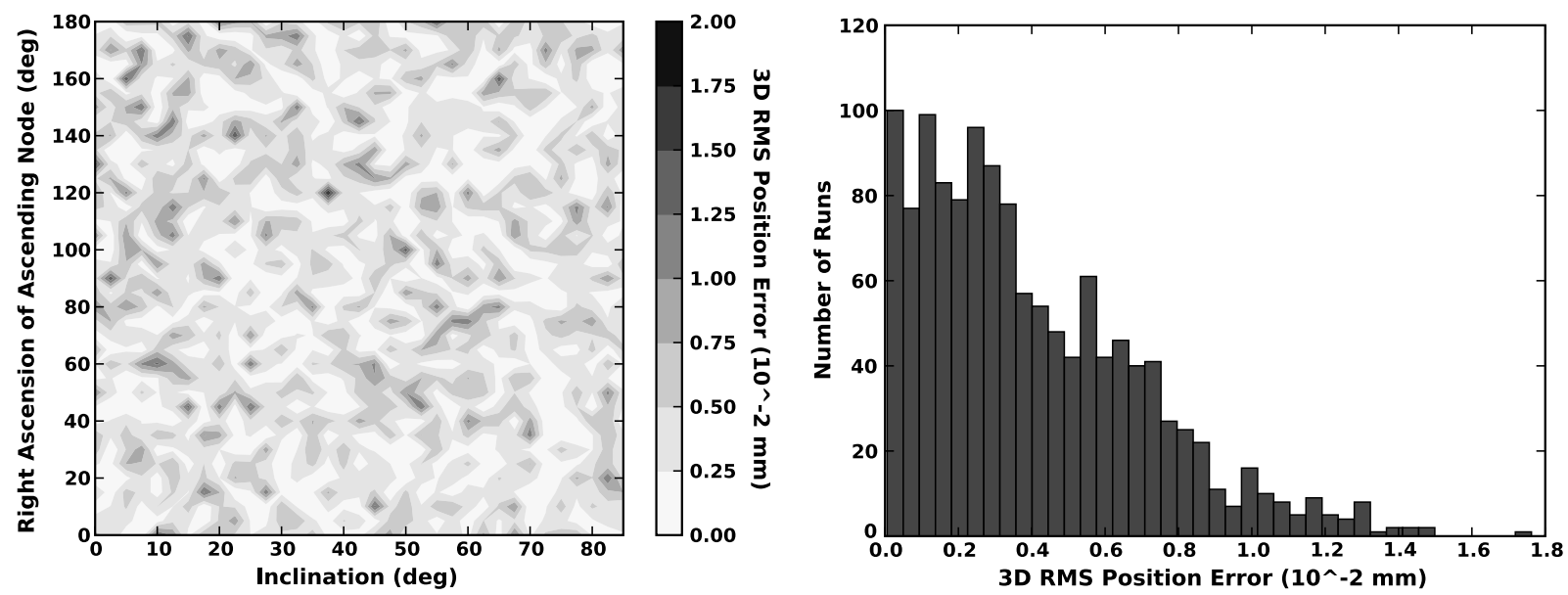

Fig. 12 Spatial distribution and histogram of the 3-D rms position differences for propagated orbits initially at $300 \mathrm{~km}$ with the CS-162 model.
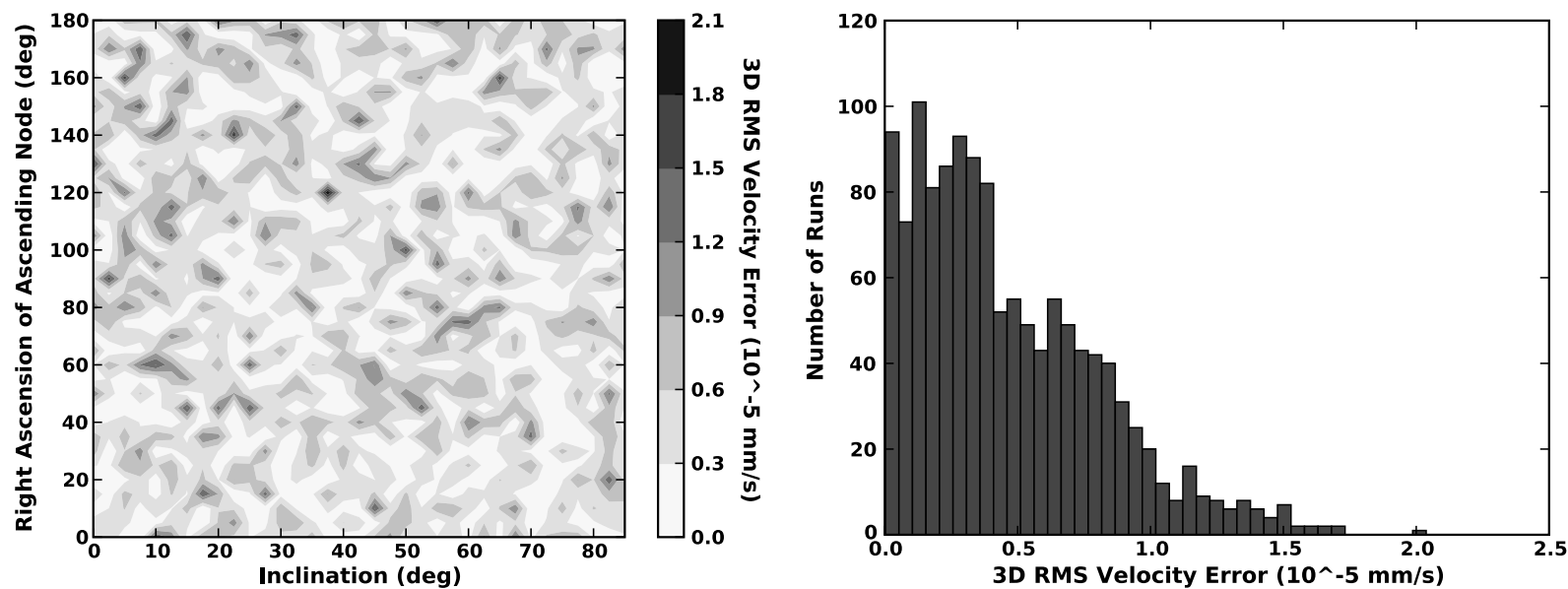

Fig. 13 Spatial distribution and histogram of the 3-D rms velocity differences for propagated orbits initially at $300 \mathrm{~km}$ with the CS-162 model. 
Table 5 Cubed-sphere state 3-D rms performance at $300 \mathrm{~km}$

\begin{tabular}{lcccccccc}
\hline \hline & \multicolumn{4}{c}{ Position, mm } & \multicolumn{3}{c}{ Velocity, mm/s } \\
\cline { 2 - 9 } Model & Min & Max & Mean & Median & Min & Max & Mean & Median \\
\hline CS-30 & $4.70 \times 10^{-5}$ & 0.0200 & 0.0042 & 0.0036 & $2.99 \times 10^{-8}$ & $2.27 \times 10^{-5}$ & $4.84 \times 10^{-6}$ & $4.11 \times 10^{-6}$ \\
CS-76 & $3.25 \times 10^{-5}$ & 0.0177 & 0.0041 & 0.0033 & $3.22 \times 10^{-8}$ & $2.04 \times 10^{-5}$ & $4.73 \times 10^{-6}$ & $3.79 \times 10^{-6}$ \\
CS-162 & $5.71 \times 10^{-5}$ & 0.0176 & 0.0040 & 0.0033 & $2.50 \times 10^{-8}$ & $2.04 \times 10^{-5}$ & $4.64 \times 10^{-6}$ & $3.80 \times 10^{-6}$ \\
\hline \hline
\end{tabular}

in the cubed-sphere model with respect to the spherical harmonics occur at lower altitudes. Additionally, the largest anomalies occur for the higher-fidelity models. At various altitudes, the differences become discretized due to machine precision and the relatively low contribution the perturbations modeled by the cubed sphere have on the overall gravity acceleration.

For the CS-162 model, there is a region below $300 \mathrm{~km}$ and around $10^{-10} \mathrm{mGal}$ at which the variations are periodic. In this case, the differences are close to the machine precision and are not affected by the grid spacing. Given the Chebyshev interpolation between shells, approximation error varies based on proximity to the nearby shells. Thus, as the altitude increases for this point in Fig. 10, the error periodically increases and decreases.

\section{Orbit-Propagation Comparisons}

Figure 11 summarizes the orbit-propagation results for the CS-30, CS-76, and CS-162 cubed-sphere models. These 3-D rms values include the state differences at all times and orbits, independent of the orbit initial conditions. Orbit position and velocity differences are on the order of fractions of $1 \mathrm{~mm}$ and of $1 \mathrm{~mm} / \mathrm{s}$, respectively. The magnitude of the orbit-propagation errors for the three models converges at $200 \mathrm{~km}$ and continues with a slight downward trend as orbit altitude increases. At low altitudes, model agreement with the base model increases with reduced fidelity of the base model, which is consistent with the gravity anomalies provided in Fig. 10. Of course, these results may be adjusted by an appropriate configuration of the cubed sphere for high-fidelity base models. However, this demonstrates that very close agreement with the base model can be achieved.

In Table 4, we present the ratio of the time required to propagate the orbit using the spherical harmonics and the cubed sphere. The computation time used in these calculations includes only the execution of the RK78 algorithm and does not include file load times or initialization. As expected, the file load time for the cubed sphere exceeds that of the spherical harmonics; however, this may be mitigated through a careful implementation. After the model reconfiguration (specifically, the increase in the degree of the polynomial interpolation between shells), the evaluation time of the model increased. The break-even point with the spherical harmonic model occurs at about degree 20. More recent results (which will be presented at a later date) demonstrate that the cubed-sphere model is faster than the spherical harmonic model for all fidelities.
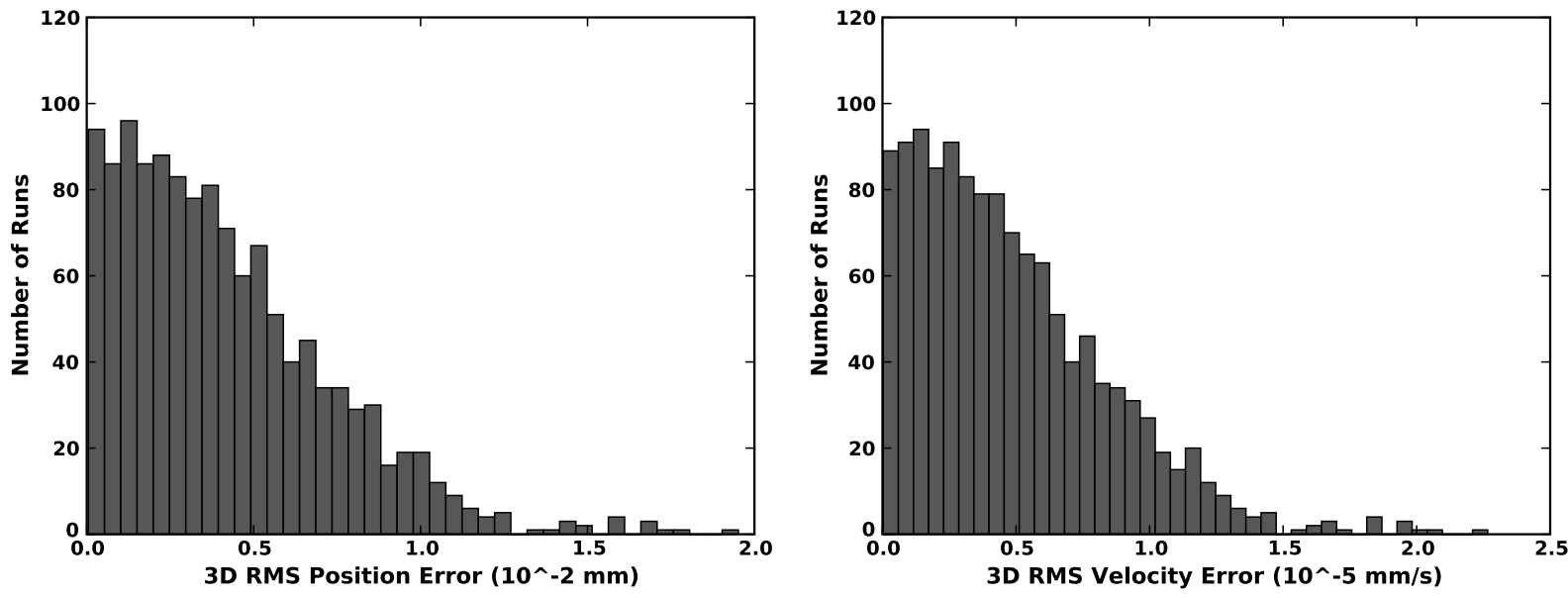

Fig. 14 Distribution of 3-D rms differences for propagated orbits initially at $300 \mathrm{~km}$ with the CS-30 model.
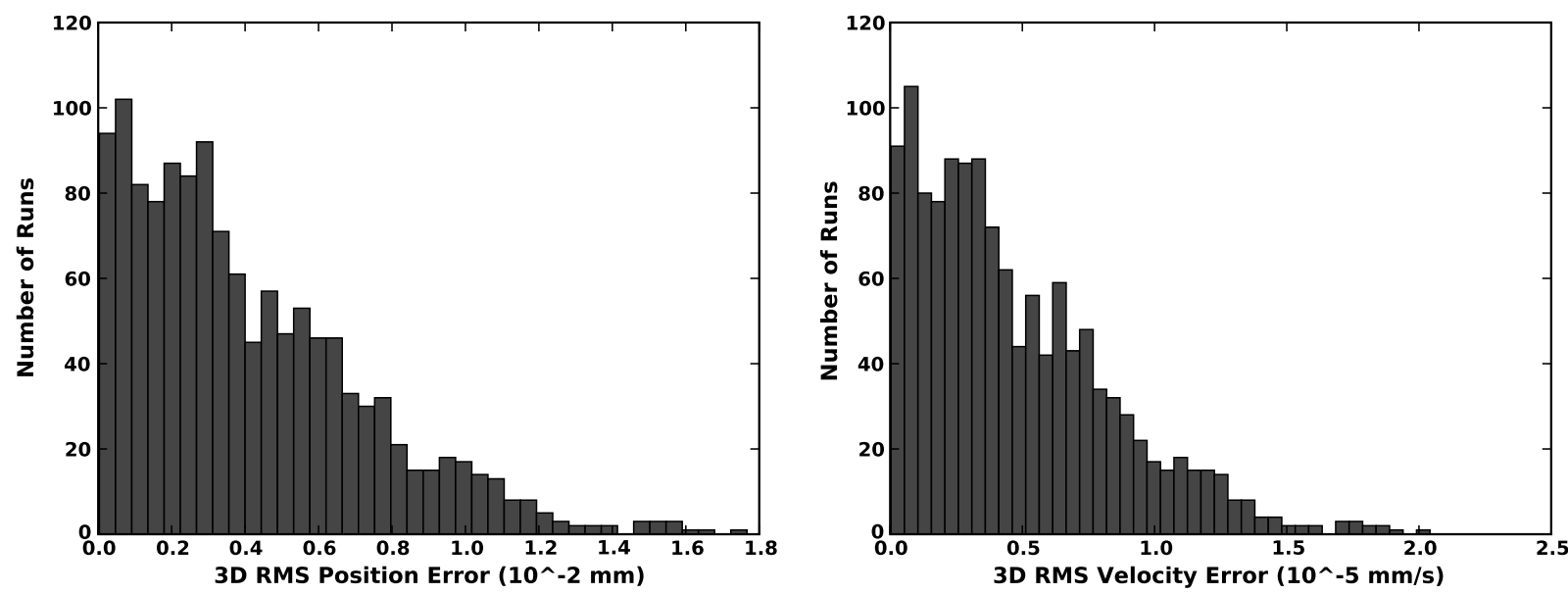

Fig. 15 Distribution of 3-D rms differences for propagated orbits initially at $300 \mathrm{~km}$ with the CS-76 model. 
Orbit-propagation performance specific to the CS-162 model at $300 \mathrm{~km}$ is illustrated in Figs. 12 and 13. There does not appear to be any trends in the errors when observing their distribution based on inclination and $\Omega$. The relatively large deviation at the $\Omega$ and inclination combination of 120 and $37.5^{\circ}$ is directly correlated with gravity anomalies in Fig. 9. Specifically, the orbit groundtrack intersects the large anomalies at approximately $28^{\circ}$ latitude and $92^{\circ}$ longitude twice during the orbit and once directly over the peak anomaly near Haiti. Other than this orbit, all others are within $0.015 \mathrm{~mm}$ of those computed via the spherical harmonics. The spatial distribution of the velocity errors roughly corresponds to that of the position errors. Table $\underline{5}$ provides statistics on the orbit state differences for all models tested.

Histograms of the propagation state errors for the CS-30 and CS76 models at $300 \mathrm{~km}$ are provided in Figs. 14 and 15, respectively. Contour plots of the errors for these models have not been included, since results are similar to those seen for the CS-162 model. Accuracy differences in the CS-30 and CS-76 models are illustrated sufficiently by the histogram plots.

Orbits with an eccentricity of 0.05 were also tested using a similar scheme, with the altitude of periapsis set at $300 \mathrm{~km}$. Results are comparable with those depicted here for all three of the cubedsphere models. Although increasing the variations in the eccentricity provides a more comprehensive comparison, larger values yield an altitude of apoapsis above $1000 \mathrm{~km}$. At these altitudes, the gravity anomalies of the cubed sphere relative to the spherical harmonics are below the machine precision (as seen in Fig. 10). Thus, higher eccentricities are not of significant interest.

To test the effects that shell boundaries have on propagated orbits, differences for a circular orbit at $358.7702 \mathrm{~km}$ were profiled. This altitude equals the altitude of the fourth primary shell. Both the integration constant and the orbit differences tests yielded results comparable with those already illustrated for a $350 \mathrm{~km}$ orbit. Thus, for the current cubed-sphere models, the primary shell boundaries do not appear to influence the orbit-propagation differences.

\section{Conclusions}

Results indicate that the cubed-sphere model closely approximates a spherical harmonic model. Orbit-propagation tests demonstrated agreements in orbit propagation on the order of fractions of a millimeter. Integration of orbits via the cubed-sphere model equivalent to a $150 \times 150$ spherical harmonic model was over 30 times faster than via the base model. As Table $\underline{4}$ indicates, the breakeven point is achieved at approximately order and degree 20 . As of this writing, tuning of the cubed sphere has been customized to the degree of the base model, with cubed-sphere evaluation time less than that of the spherical harmonics for all configurations.

The new model configuration presented here greatly reduced variations in the integration constant, with changes consistent between the cubed sphere and the spherical harmonic models. The agreement between the models is reduced below $200 \mathrm{~km}$. In some cases, the cubed-sphere model actually performs better than the corresponding spherical harmonic model, probably due to the random nature of fluctuations in the integration constant near the limit of the machine precision. Gravity anomalies were also reduced and are now within $10^{-6} \mathrm{mGal}$ for all altitudes above the Earth and less than $10^{-7} \mathrm{mGal}$ for altitudes at or above $300 \mathrm{~km}$ when using the current configuration.

As of this writing, characterization of the accuracy of moon-based models is still in progress. Initial orbit-propagation results demonstrated that additional tuning of the cubed sphere for lunar applications is required. This is due to mass concentrations caused by asteroid impacts creating relatively extreme gravity variations. Unlike Earth-centered orbits, lower-altitude orbits are possible at the moon, since atmospheric drag is not an issue. Thus, tuning of lunar models should incorporate accurate representations to much lower altitudes. When the ratio of the primary body radius to the orbit radius is considered, $300 \mathrm{~km}$ is equivalent to a lunar orbit with an altitude of approximately $82 \mathrm{~km}$. Thus, some additional tuning is required to improve model performance at the minimum desired altitude of $50 \mathrm{~km}$ at the moon.

Future research includes incorporating the cubed sphere with the orbit determination process for both Earth- and moon-based missions. Even though second derivatives are not currently included in the cubed sphere, integration with nonlinear filters, such as the unscented Kalman, is rather straightforward.

\section{Appendix: Basis Splines}

A simple way to introduce basis splines (or B-splines) is to define them as the convolution:

$$
B_{m}(x)=\left(B_{m-1} * B_{0}\right)(x)
$$

where

$$
B_{0}(x)= \begin{cases}1, & |x| \leq \frac{1}{2} \\ 0, & \text { otherwise }\end{cases}
$$

Thus, $B_{m}$ is a piecewise polynomial of degree $m$. On taking the Fourier transform of $B_{0}$,

$$
\int_{-\infty}^{+\infty} B_{0}(x) e^{-2 \pi i x \xi} \mathrm{d} x=\frac{\sin \pi \xi}{\pi \xi}
$$

we obtain

$$
\int_{\infty}^{+\infty} B_{m}(x) e^{-2 \pi i x \xi} \mathrm{d} x=\left(\frac{\sin \pi \xi}{\pi \xi}\right)^{m+1}
$$

We only consider B-splines of odd degree and note that in such cases the $m$ th degree B-spline is nonzero only in the interval $[-(m+1) / 2,(m+1) / 2]$. For our purposes, we use a periodized version of B-splines on the interval $[0,1]$. Subdividing $[0,1]$ into $N=2^{k}$ subintervals, where $N \geq m+1$ (in practice, $N \gg m+1$ ), we consider the basis of B-splines on this subdivision:

$$
\left\{B_{m}(N x-j)\right\}_{j=0,1, \ldots, 2^{k}-1}
$$

Let us consider a function $g(x)$ that may be written as

$$
g(x)=\sum_{j=0}^{N-1} \alpha_{j} B_{m}(N x-j)
$$

Instead of using the basis of B-splines, we may also write the same function as

$$
g(x)=\sum_{j=0}^{N-1} \gamma_{j} L_{m}(N x-j)
$$

where $L_{m}$ are interpolating splines of a given degree $m$; that is,

$$
L_{m}(l)=\delta_{l, 0}
$$

$l$ is an integer, and $\delta_{i, j}$ is the Kronecker delta function. The definitions of interpolating splines implies that the coefficients in Eq. (A6) are, in fact, the values of the function $g(x)$ on the lattice:

$$
\gamma_{l}=g(l / N)
$$

In our problem, we are given the values $\gamma_{l}=g(l / N)$ and we need to find the coefficients $\alpha_{j}$ in Eq. (A5). We have

$$
\int_{0}^{1} B_{m}(N x-j) e^{-2 \pi i x n} \mathrm{~d} x=\frac{1}{N} \hat{B}_{m}\left(\frac{n}{N}\right) e^{-2 \pi i j n / N}
$$

and computing the Fourier coefficients of $g$ in Eq. (A5), we obtain

$$
\hat{g}_{n}=\left(\frac{1}{N} \sum_{j=0}^{N-1} \alpha_{j} e^{-2 \pi i j n / N}\right) \hat{B}_{m}\left(\frac{n}{N}\right)=\hat{\alpha}_{n} \hat{B}_{m}\left(\frac{n}{N}\right)
$$


Similarly, we compute the Fourier coefficients of $g$ in terms of interpolating splines:

$$
\hat{g}_{n}=\left(\frac{1}{N} \sum_{j=0}^{N-1} \gamma_{j} e^{-2 \pi i j n / N}\right) \hat{L}_{m}\left(\frac{n}{N}\right)=\hat{\gamma}_{n} \hat{L}_{m}\left(\frac{n}{N}\right)
$$

The B-splines and the interpolating splines are related by (see, for example, [16])

$$
\hat{L}_{m}\left(\frac{n}{N}\right)=\frac{\hat{B}_{m}\left(\frac{n}{N}\right)}{a\left(\frac{n}{N}\right)}
$$

where

$$
a(\omega)=\sum_{j \in \mathbb{Z}}\left|\hat{B}_{m}(\omega+j)\right|^{2}
$$

It may be shown that $a$ is a trigonometric polynomial (see, for example, [16]),

$$
\sum_{j \in \mathbb{Z}}\left|\hat{B}_{m}(\omega+j)\right|^{2}=\sum_{l=-m}^{m} B_{2 m+1}(l) e^{-2 \pi i l \omega}
$$

thus simplifying the evaluation of $a$. Finally, substituting Eq. ( $\underline{\mathrm{A} 12})$ into Eq. (A11), we get

$$
\hat{g}_{n}=\hat{\gamma}_{n} \frac{\hat{B}_{m}\left(\frac{n}{N}\right)}{a\left(\frac{n}{N}\right)}
$$

which implies

$$
\hat{\alpha}_{n}=\frac{\hat{\gamma}_{n}}{a\left(\frac{n}{N}\right)}
$$

In other words, applying the discrete Fourier transform to the data values $\gamma_{l}=g(l / N)$, scaling by the factor $1 / a(n / N)$ and applying the inverse discrete Fourier transform, we obtain the coefficients $\alpha_{j}$ in Eq. (A5). The two-dimensional case is a straightforward extension, where

$$
\hat{\alpha}_{k, l}=\frac{\hat{\gamma}_{k, l}}{a\left(\frac{k}{N}\right) a\left(\frac{l}{N}\right)}
$$

\section{Acknowledgments}

A large portion of this research was funded by the NASA Graduate Student Researchers Program (NASA/GSRP) through the NASA Johnson Space Center. Gregory Beylkin's research is supported by U.S. Air Force Office of Scientific Research grant FA9550-07-10135. The authors would like to thank Keric Hill, formerly of the Colorado Center for Astrodynamics Research, who wrote the early versions of TurboProp, and the anonymous reviewers for their time and suggestions.

\section{References}

[1] Konopliv, A. S., Asmar, S. W., Carranza, E., Sjogren, W., and Yuan, D., "Recent Gravity Models as a Result of the Lunar Prospector Mission," Icarus, Vol. 150, No. 1, 2001, pp. 1-18. doi:10.1006/icar.2000.6573

[2] Beylkin, G., and Cramer, R., "Toward Multiresolution Estimation and Efficient Representation of Gravitational Fields," Celestial Mechanics and Dynamical Astronomy, Vol. 84, 2002, pp. 87-104. doi:10.1023/A:1019941111529

[3] Junkins, J. L., "Investigation of Finite-Element Representations of the Geopotential," AIAA Journal, Vol. 14, No. 6, June 1976, pp. 803-808. doi: $10.2514 / 3.61420$

[4] Engels, R. C., and Junkins, J. L., "Local Representation of the Geopotential by Weighted Orthonormal Polynomials," Journal of Guidance and Control, Vol. 3, No. 1, Jan.-Feb. 1980, pp. 55-61. doi: 10.2514/3.55947

[5] Colombi, A., Hirani, A. N., and Villac, B. F., "Adaptive Gravitational Force Representation for Fast Trajectory Propagation Near Small Bodies," Journal of Guidance, Control, and Dynamics, Vol. 31, No. 4, July-August 2008, pp. 1041-1051. doi: $10.2514 / 1.32559$

[6] Hujsak, R., "Gravity Acceleration Approximation Functions," Advances in the Astronautical Sciences, Vol. 93, No. 1, 1996, pp. 335-349.

[7] Schmidt, M., Fengler, M., Mayer-Gurr, T., Eicker, A., Kusche, J., Sanchez, L., and Han, S., "Regional Gravity Modeling in Terms of Spherical Base Functions," Journal of Geodesy, Vol. 81, No. 1, 2007, pp. 17-38. doi:10.1007/s00190-006-0101-5

[8] Fengler, M. J., Michel, D., and Michel, V., "Harmonic Spline-Wavelets on the 3-Dimensional Ball and Their Application to the Reconstruction of the Earth's Density Distribution from Gravitational Data at Arbitrarily Shaped Satellite Orbits," ZAMM: Journal of Applied Mathematics and Mechanics, Vol. 86, No. 11, Nov. 2006, pp. 856-873. doi:10.1002/zamm.200510277

[9] Mautz, R., Scharin, B., Shum, C. K., and Han, S.-C., Earth Observation with CHAMP, Results from Three Years in Orbit, Springer, Berlin, 2004, pp. 230-236.

[10] deBoor, C., A Practical Guide to Splines, Vol. 27, Applied Mathematical Sciences, Springer, New York, 2001, pp. 20-21.

[11] Beylkin, G., and Monzon, L., "Approximation by Exponential Sums Revisited," Applied and Computational Harmonic Analysis (accepted for publication).

[12] Ahrens, C., and Beylkin, G., "Rotationally Invariant Quadratures for the Sphere," Proceedings of the Royal Society of London A, Vol. 465, No. 2110 , Oct. 2009, pp. 3103-3125. doi:10.1098/rspa.2009.0104

[13] Tapley, B., Ries, J., Bettadpur, S., Chambers, D., Cheng, M., Condi, F., Gunter, B., Kang, Z., Nagel, P., Pastor, R., Pekker, T., Poole, S., and Wang, F., "GGM02-An Improved Earth Gravity Field Model from GRACE," Journal of Geodesy, Vol. 79, 2005, pp. 467-478. doi:10.1007/s00190-005-0480-z

[14] Hill, K., and Jones, B. A., "TurboProp Version 3.3," Colorado Center for Astrodynamics Research, Univ. of Colorado, Boulder, CO, Sept. 2008, http://ccar.colorado.edu/geryon/papers/TurboProp_3_3_ Manual.pdf, [retrieved 12 Dec. 2009].

[15] Bond, V. R., and Allman, M. C., Modern Astrodynamics, Princeton Univ. Press, Princeton, NJ, 1996, p. 138.

[16] Chui, C. K., An Introduction to Wavelets, Vol. One of Wavelet Analysis and Its Applications, 1st ed., Academic Press, Boston, 1992, pp. 89, $110-112$. 